\title{
Diaphragm thickness and inspiratory strength in patients with Duchenne muscular dystrophy
}

\author{
P F De Bruin, J Ueki, A Bush, Y Khan, A Watson, N B Pride
}

\begin{abstract}
Background - There is little information on the morphometric characteristics of the diaphragm in patients with Duchenne muscular dystrophy.

Methods - The thickness of the diaphragm was measured at the zone of apposition using $B$ mode ultrasonography in 10 boys with Duchenne muscular dystrophy of mean (SD) age $10.3(1.3)$ years and 12 normal controls of mean (SD) age 11.3 (2.0) years during relaxation (DiTrelax) and during maximum effort inspiratory manoeuvres (DiTPImax) at functional residual capacity.

Results - DiTrelax was greater in the patients with Duchenne muscular dystrophy $(1.74(0.21) \mathrm{mm})$ than in controls $(1.48(0.20) \mathrm{mm})$, mean difference $(95 \%$ CI) $0.26(0.08$ to 0.44$)$, despite considerable impairment of maximum effort inspiratory mouth pressure (PImax) (patients with Duchenne muscular dystrophy -37 (8) $\mathrm{cm} \mathrm{H}_{2} \mathrm{O}$, controls -80 (33) $\left.\mathrm{cm} \mathrm{H}_{2} \mathrm{O}\right)$, mean difference $(95 \% \mathrm{CI})$ 43 (65 to 20). During a PImax manoeuvre, compared with measurements taken during relaxation, the diaphragm thickened 1.6 times in patients with Duchenne muscular dystrophy and 2.3 times in controls $\begin{array}{lll}(\text { DiTPImax } 2.62 & (0.7) \mathrm{mm} \text { and } 3.5\end{array}$ $(0.85) \mathrm{mm}$, respectively), mean difference $(95 \%$ CI $)-0.88(-1.58$ to -0.18$)$.

Conclusions - Resting diaphragm thickness is increased in young patients with Duchenne muscular dystrophy with impaired respiratory muscle force. This finding could be analogous to the pseudohypertrophy that is observed in some limb muscle groups.

(Thorax 1997;52:472-475)
\end{abstract}

Keywords: diaphragm thickness, inspiratory muscle strength, ultrasonography.

Ventilatory failure is the commonest cause of death in Duchenne muscular dystrophy. ${ }^{1}$ Progressive respiratory muscle weakness starts early ${ }^{2}$ but, because hypercapnia is usually preterminal, it has been suggested that the diaphragm might be selectively spared until a late phase of the disease. ${ }^{3}$

Ultrasonography has proved useful for the study of anatomical characteristics of many muscle groups. ${ }^{4}$ Recently, high resolution ultrasound scanning has been used to assess diaphragm thickness during tidal breathing and during relaxation in normal subjects. ${ }^{56}$ Using B mode ultrasonography we have imaged the costal portion of the diaphragm both at rest and during maximum voluntary contractions in normal subjects. ${ }^{7}$ In this paper we have made ultrasonographic measurements of diaphragm thickness in young patients with Duchenne muscular dystrophy and age matched normal boys.

\section{Methods}

SUBJECTS

Ten boys with Duchenne muscular dystrophy (mean age 10.3, range $7-12$ years, mean body weight $103.4 \%$ of ideal, mean height $97.3 \%$ of predicted values) who regularly attended the Hammersmith Hospital Muscle Clinic took part in the study. The upper age limit was set on the basis of preliminary ultrasound studies carried out in four boys aged 14-17 years with Duchenne muscular dystrophy which allowed no clear visualisation of the diaphragm (see discussion). All patients had progressive, predominantly proximal, muscular weakness without sensory abnormalities which had developed before the age of five and enlarged calves or Achilles tendon contractures, or both. Two boys were confined to a wheel chair. They all had serum creatine kinase levels, electromyographic and muscle biopsy findings consistent with the diagnosis of Duchenne muscular dystrophy, and had no respiratory symptoms. Twelve normal boys (mean age 11.3 , range $6-12$ years, mean body weight $103.0 \%$ of ideal, mean height $98.6 \%$ of predicted values) were studied as controls.

\section{MEASUREMENTS}

Standing height was recorded without shoes; in three boys with Duchenne muscular dystrophy it was estimated from arm span. Body weight was recorded with indoor clothes. Results were compared with values derived from the weight-for-age and height-for-age curves from the National Center for Health Statistics and Center for Disease Control and expressed as a percentage of the median for the reference population using Epi Info, version 5. Forced expiratory volume in one second $\left(\mathrm{FEV}_{1}\right)$, forced 

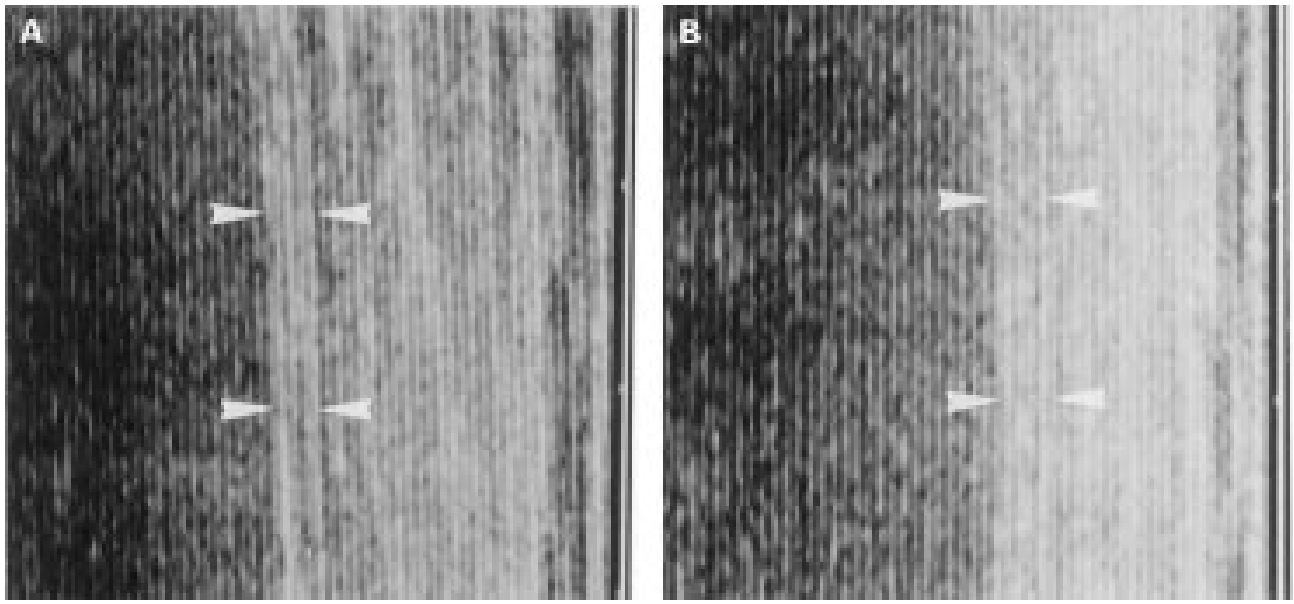

Figure 1 Ultrasonograms of the right hemidiaphragm at the apposition zone during relaxation at functional residual capacity in (A) a 13 year old normal boy and (B) a 10 year old boy with Duchenne muscular dystrophy. Arrows indicate the outer edges of the peritoneal (left) and pleural (right) membranes lining the diaphragm. There is increased echogenicity of the structures of the intercostal space overlying the costal diaphragm.

vital capacity (FVC), and peak expiratory flow (PEF) were recorded using a Fukuda Sangyo spiro-analyser (ST250). Predicted values were derived from Cotes. ${ }^{8}$

Real-time movement of the diaphragm was recorded by $\mathrm{B}$ mode ultrasonography. A $7.5 \mathrm{MHz}$ ultrasound linear probe (PLE 705S Toshiba Medical System) was held perpendicular to the chest wall in the ninth or tenth right intercostal space between the antero- and mid-axillary lines. If the angle of incidence was changed significantly by movement of the transducer head the returning ultrasound signal of the diaphragm became distorted or was lost altogether, as reported previously. ${ }^{57}$ The diaphragm was observed bounded by two clear outer parallel lines corresponding to the pleural and peritoneal membranes with an irregular bright layer of connective tissue and vessels within the muscle layer (fig 1). Diaphragm thickness was measured from the middle of the pleural to the middle of the peritoneal line. The shortest distance that could be resolved between the two lines using this system was $0.3 \mathrm{~mm}$, considerably less than the thickness of the normal relaxed diaphragm. Measurements of thickness were made to the nearest $0.1 \mathrm{~mm}$ with calipers. On each ultrasound image two measurements of thickness were made by a single experienced observer at $0.5-2.0 \mathrm{~cm}$ below the costophrenic sinus and the results were averaged.

Initially three separate measurements of resting diaphragm thickness at FRC were taken while the subject was sitting comfortably, wearing a noseclip and breathing through a conventional mouthpiece, and the mean value (DiTrelax) of the measurements made on the three ultrasound images was used for analysis. In a set of six measurements the thickness was very consistent; in 19 of the boys the maximum difference was $0.1 \mathrm{~mm}$ or less and in the remaining three boys it was $0.2 \mathrm{~mm}$. The subject was then asked to perform a maximum effort inspiratory manoeuvre against a closed airway at FRC for two seconds while the diaphragm thickness was assessed by ultrasonography. The pressure generated at the mouthpiece was recorded using a variable inductance differential pressure transducer (HP 267B) with a small internal volume and linear response over the range of 200 to $-200 \mathrm{~cm} \mathrm{H}_{2} \mathrm{O}$. A small leak was introduced into the system to ensure glottal patency and to avoid the use of cheek muscles. The procedure was repeated at least three times. Maximum effort inspiratory mouth pressure (PImax) was taken as the most negative pressure sustained for at least one second and the thickness of the diaphragm on the ultrasound image obtained during this manoeuvre (DiTPImax) was selected for analysis. The increase in diaphragm thickness during this maximum effort inspiratory manoeuvre was calculated as the thickening ratio (TRDi):

$$
\mathrm{TRDi}=\frac{\text { DiTPImax }}{\text { DiTrelax }}
$$

Maximum effort expiratory mouth pressure (PEmax) was measured near TLC and the best of three technically satisfactory manoeuvres sustained for at least one second was selected for analysis. Normal values for mouth pressures were taken from Wilson and coworkers. ${ }^{9}$

Written informed consent was obtained in all cases and the protocol was approved by the local research ethics committee.

\section{ANALYSIS OF DATA}

Spearman rank correlation coefficients $\left(r_{\mathrm{S}}\right)$ were used to define correlations. The magnitude of the correlations between PImax and age, body weight, DiTrelax, and TRDi and between DiTrelax and age, height, and body weight was assessed by linear regression analysis. Changes in diaphragm thickness and maximum inspiratory and expiratory mouth pressures were compared by the $t$ test; mean values are given with $95 \%$ confidence intervals (95\% CI). Group results are expressed as mean (SD) values. 
Table 1 Mean (SD) values of respiratory muscle strength and thickness assessed by ultrasonography in patients with Duchenne muscular dystrophy (DMD) and normal subjects

\begin{tabular}{lcccr}
\hline & $\begin{array}{l}\text { DMD } \\
(n=10)\end{array}$ & $\begin{array}{l}\text { Normal }(N) \\
(n=12)\end{array}$ & $\begin{array}{l}\text { Mean difference } \\
D M D-N(95 \% \text { CI })\end{array}$ & $p$ value \\
\hline PImax (cm H $\left.\mathrm{H}_{2} \mathrm{O}\right)$ & $-37(8)$ & $-80(33)$ & $43(65$ to 20$)$ & $<0.001$ \\
PImax (\% predicted) & $58.0(17.4)$ & $107.2(37.9)$ & $-49.2(-76$ to -22$)$ & 0.001 \\
DiTrelax (mm) & $1.74(0.21)$ & $1.48(0.20)$ & $0.26(0.08$ to 0.44$)$ & 0.007 \\
DiTPImax (mm) & $2.62(0.70)$ & $3.50(0.85)$ & $-0.88(-1.58$ to -0.18$)$ & 0.017 \\
TRDi & $1.6(0.5)$ & $2.3(0.4)$ & $-0.7(-1.1$ to -0.30$)$ & 0.001 \\
PEmax (cm $\left.\mathrm{H}_{2} \mathrm{O}\right)$ & $41(12)^{*}$ & $94(49)$ & $-53(-90$ to -16$)$ & 0.007 \\
PEmax (\% predicted) & $43.5(13.7)^{*}$ & $95.1(44.0)$ & $-51.5(-85.5$ to -17.5$)$ & 0.004 \\
\hline
\end{tabular}

PImax $=$ maximum effort inspiratory mouth pressure; DiTrelax = resting diaphragm muscle thickness at FRC; DiTPImax = diaphragm muscle thickness during PImax manoeuvre; TRDi = diaphragm thickening ratio; PEmax $=$ maximum effort expiratory pressure.

pressure.

\section{Results}

Mean (SD) $\mathrm{FEV}_{1}(1.45$ (0.27) $1 ; 77.0(14.8) \%$ predicted), FVC (1.52 (0.29) $1 ; 65.8(12.0) \%$ predicted), and PEF (2.66 (0.66) $1 / \mathrm{s} ; 55.7$ $(12.8) \%$ predicted) were all significantly reduced in boys with Duchenne muscular dystrophy compared with normal boys $(p<0.025$ for all comparisons). $\mathrm{FEV}_{1} / \mathrm{FVC} \%$ was significantly higher in boys with Duchenne muscular dystrophy $(95.8(4.9) \%)$ than in normal boys (89.3 (5.4)\%; p<0.001).

PImax was less negative in patients with Duchenne muscular dystrophy $(-37(8) \mathrm{cm}$ $\mathrm{H}_{2} \mathrm{O}$ ) than in controls $\left(-80\right.$ (33) $\mathrm{cm} \mathrm{H}_{2} \mathrm{O}$ ); mean difference ( $95 \% \mathrm{CI}) 43$ (65 to 20 ), $\mathrm{p}<0.001$ (table 1). Significant negative correlations were found between PImax and age $\left(r_{\mathrm{S}}=0.431 ; \mathrm{p}=0.02\right)$ and PImax and body weight $\left(r_{\mathrm{S}}=0.486 ; \mathrm{p}=0.018\right)$ in the control group. Pemax was lower in patients with $\mathrm{Du}-$ chenne muscular dystrophy $\left(41\right.$ (12) $\mathrm{cm} \mathrm{H}_{2} \mathrm{O}$ ) than in control subjects $\left(94(49) \mathrm{cm} \mathrm{H}_{2} \mathrm{O}\right.$ ), mean difference $(95 \% \mathrm{CI})-53(-90$ to -16$)$, $\mathrm{p}=0.007$.

Despite reduced inspiratory force, patients with Duchenne muscular dystrophy showed a greater mean DiTrelax $(1.74(0.21) \mathrm{mm})$ than controls $(1.48 \quad(0.20) \mathrm{mm}$, mean difference (95\% CI) 0.26 (0.08 to 0.44$), p=0.007$, (table 1; fig 2). During maximum effort inspiratory manoeuvres at FRC there was an average thick-

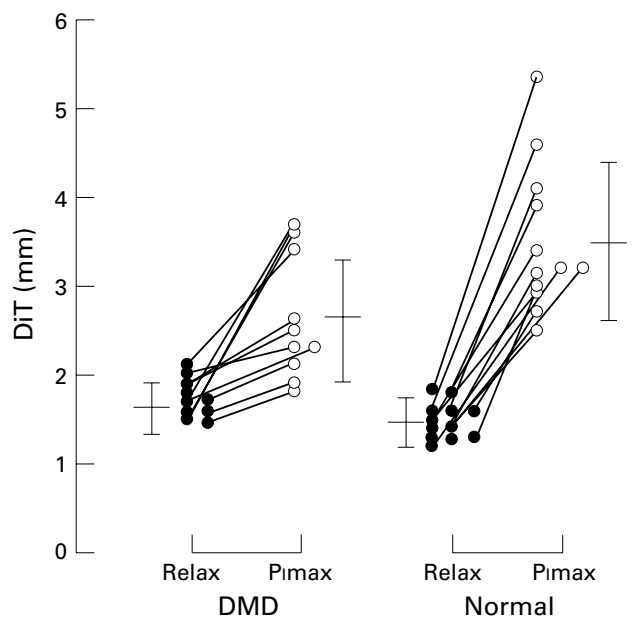

Figure 2 Diaphragm thickness (DiT) at functional residual capacity during relaxation and during a maximum inspiratory effort (PImax) in 10 boys with Duchenne muscular dystrophy (DMD) and 12 normal boys. Bars indicate mean values $\pm 1 S D$. ening of the diaphragm of 1.6 times in patients with Duchenne muscular dystrophy and 2.3 times in controls compared with measurements taken during relaxation at a similar lung volume (mean DiTPimax $2.62(0.70) \mathrm{mm}$ and 3.5 $(0.85) \mathrm{mm}$, respectively, mean difference $(95 \%$ CI) $-0.88(-1.58$ to -0.18$), p=0.017)$. DiTrelax showed a significant positive correlation with age $\left(r_{\mathrm{S}}=0.378 ; \mathrm{p}=0.035\right)$ and body weight $\left(r_{\mathrm{S}}=0.655 ; \mathrm{p}=0.001\right)$ and a negative correlation with PImax $\left(r_{\mathrm{S}}=0.483 ; \mathrm{p}=\right.$ 0.020 ) in normal subjects but not in patients with Duchenne muscular dystrophy. Thickening of the diaphragm (TRDi) was significantly negatively correlated to PImax only in the normal group $\left(r_{\mathrm{S}}=0.338 ; \mathrm{p}=0.047\right)$.

\section{Discussion}

These results show that resting diaphragm muscle thickness (DiTrelax) is increased in patients with Duchenne muscular dystrophy below the age of 12 years with significant reductions in spirometric measurements and maximum effort mouth pressures.

In preliminary studies using ultrasonography carried out in four patients with Duchenne muscular dystrophy older than 14 years echogenicity of the overlying intercostal muscles was increased and the pleural and peritoneal surfaces of the diaphragm were poorly defined so that we could not obtain reliable thickness measurements. In these four patients diaphragm echogenicity was increased, resembling the bright and speckled echo pattern found in limb muscles in patients with Duchenne muscular dystrophy which has been attributed to deposition of adipose and connective tissue. ${ }^{1011}$

PImax was considerably impaired in the boys with Duchenne muscular dystrophy, supporting previous reports of an early involvement of the respiratory muscles in this illness. ${ }^{12} \mathrm{Al}-$ though PImax assesses the overall force produced by the inspiratory muscles, maximum activation of the diaphragm is usually achieved with this manoeuvre, at least in normal subjects. ${ }^{12}$ Despite the impairment of PImax, DiTrelax was significantly greater in the patients with Duchenne muscular dystrophy $(1.74(0.21) \mathrm{mm})$ than in the controls $(1.48$ $(0.20) \mathrm{mm}$ ). In normal boys the mean DiTrelax was significantly thinner than was found in our earlier study ${ }^{7}$ of 13 normal adult men of average age 37 years $(1.68(0.21) \mathrm{mm}$, mean difference $(95 \% \mathrm{CI})-0.2(-0.34$ to -0.03$), \mathrm{p}=0.023)$ 
and was significantly correlated to age, body weight, and inspiratory muscle force. Diaphragm thickening during maximum effort inspiration (TRDi) in patients with Duchenne muscular dystrophy was less than was observed in controls. This index has previously been shown to be a good indicator of inspiratory muscle strength in normal adults. ${ }^{7}$

To our knowledge, no other studies of diaphragm thickness in patients with Duchenne muscular dystrophy have been published. Increased size of the calves and certain other limb muscle groups, also known as pseudohypertrophy, is a well known feature of Duchenne muscular dystrophy. Pseudohypertrophy is progressive in the early stages of the disease but most of the muscles eventually decrease in size, except for the gastrocnemii and, to a lesser extent, the quadriceps and deltoids. The enlarged muscles are less strong and more hypotonic than healthy muscles of the same size. ${ }^{13}$ Infiltration of connective tissue and deposition of fat appears to be the cause for the increased size of calf muscles but these changes are not closely related to age or severity of disease. ${ }^{14}$ Similar changes have been found in intercostal muscle biopsy specimens from four patients with Duchenne muscular dystrophy ${ }^{15}$ but, unfortunately, no studies of diaphragm muscle composition in Duchenne muscular dystrophy are available.

We conclude that diaphragm thickness is increased in young patients with Duchenne muscular dystrophy with impaired respiratory muscle force. This finding may be analogous to the pseudohypertrophy that occurs in some limb muscles during the course of this disease.
PFDB was supported by the Conselho Nacional de Desenvolvimento Científico e Tecnológico-CNPq of Brazil and JU by the Glaxo International Scholarship Charitable Fund. The author are grateful to Professor V Dubowitz for access to patient under his care, to the staff and boys of the Burlington Danes School for allowing them to study control subjects, and to Dr A Manzur for his help and support.

1 Inkley CR, Oldenburg FC, Vignos PL. Pulmonary function in Duchenne muscular dystrophy related to stage of disin Duchenne muscular dystrophy

2 Smith PEM, Calverley PMA, Edwards RHT, Evans GA Campbell EJM. Practical problems in the respiratory care of patients with muscular dystrophy. N Engl F Med 1987; 316:1205-10

3 Newsom-Davis J. The respiratory system in muscular dystrophy. Br Med Bull 1980;36:135-8.

4 Young A, Stokes M, Crowe M. Size and strength of quadriceps muscles of old and young women. Eur F Clin Invest 1984;14:282-7.

5 Wait JL, Nahormek PA, Yost WT, Rochester DF. Diaphragmatic thickness-lung volume relationship in vivo. $\mathcal{F}$ Appl Physiol 1989;67:1560-8.

6 Taniguchi N, Miyakoda J, Itoh K, Fukui J, Nakamura M, Suzuki O. Ultrasonographic images of the diaphragm and respiratory changes on their thickness. $\mathcal{F p}_{p} \mathcal{F}$ Med Ultrasonics 1991;18:93-8.

7 Ueki J, De Bruin PF, Pride NB. The in vivo assessment of diaphragm thickness by ultrasound. Thorax 1995:50. of diaphragn $1157-61$.

8 Cotes JE. Lung function: assessment and application in medicine. 5th ed. Oxford: Blackwell Scientific Publications, 1993. 5 th ed. Oxf

9 Wilson SH, Cooke NT, Edwards RHT, Spiro SG. Predicted normal values for maximal respiratory pressures in caucasian adults and children. Thorax 1984;39:535-8.

10 Heckmatt JZ, Dubowitz V, Leeman S. Detection of pathological change in dystrophic muscle with B-scan ultrasound imaging. Lancet 1980;i:1389-90.

11 Heckmatt JZ, Pier N, Dubowitz V. Real-time ultrasound imaging of muscles. Muscle Nerve 1988;11:56-65.

12 Gandevia SC, McKenzie DK, Plassman BL. Activation of human respiratory muscles during different voluntary manoeuvres. F Physiol (Lond) 1990;428:387-403.

13 Adams RD, Victor M. The muscular dystrophies. In: Adams $\mathrm{RD}$, Victor M, eds. Principles of neurology. 4 th ed. New York: McGraw-Hill, 1989: 1117-32.

14 Jones DA, Round JM, Edwards RHT, Grindwood SR, Tofts Jon DA, Round JM, Edwards RHT, Grindwood SR, Tofts
PS. composition of the calf and quadriceps muscles in Duchenne muscular dystrophy. 7 Neurol Sci 1983;60:307-22.

15 Stern LZ, Payne CM, Gruener R, Anderson RM, Hannapel LK. Intercostal muscle biopsy in neuromuscular disease; histochemical and electron microscopic studies. F Neuro Neurosurg Psychiatry 1975;38:900-10. 